\title{
New myeloperoxidase detection system based on enzyme-catalysed oxidative synthesis of a dye for paper-based diagnostic devices
}

\author{
Arnau Bassegoda ${ }^{\dagger}$, Guillem Ferreres ${ }^{\dagger}$, Sílvia Pérez-Rafael ${ }^{\dagger}$, Juan Torrent-Burgués \\ and Tzanko Tzanov ${ }^{*}, \dagger$. \\ †Grup de Biotecnologia Molecular i Industrial, Department of Chemical Engineering, \\ Universitat Politècnica de Catalunya, Rambla Sant Nebridi 22, Terrassa 08222, Spain. \\ E-mail: tzanko.tzanov@upc.edu \\ ${ }^{*}$ Corresponding author
}

\begin{abstract}
The severity and cost of wound infections strongly demands for simple and fast methods for wound infection determination. Point-of-care testing devices play a crucial role in order to achieve a fast diagnosis and early treatment. Myeloperoxidase (MPO)* enzyme, detected in fluids of infected wounds has been postulated as a suitable biomarker for wound diagnostics. Here we present a new system for MPO detection, based on enzyme-catalysed oxidative synthesis of a dye that can be incorporated into paper-based point of care devices. Visual MPO detection has been achieved through the use of phenylenediamine, a common colourless hair dye precursor. MPO oxidation of these compounds yielded bright coloured products distinguishable from the colour of the wound environment. Immobilisation of the MPO substrates on paper strips was achieved through in situ interaction of the oxidised coloured product with branched polyethyleneimine. The colour reaction of the immobilized substrates, detectable by naked eye, responds to the MPO levels present in infected wound fluids revealing an easy system for incorporation of MPO detection in paper based diagnostic devices.
\end{abstract}

*Abbreviations: Myeloperoxidase (MPO), point of care testing (PoCT), hypochlorous acid $(\mathrm{HClO})$, glucose oxidase (GOX), $p$-phenilenediamine (pPD), $m$-phenilenediamine $(\mathrm{mPD})$ and $o$ - phenilenediamine (oPD), polyethyleneimine branched (PEI) 


\section{Keywords}

Myeloperoxidase, chronic wounds, diagnostics, dye

\section{Introduction}

Chronic wounds represent a challenge to wound care professionals and consume a great deal of healthcare resources around the globe. The severity and cost of wound infections increase dramatically the longer they remain untreated. The resulting pain, impairment and social isolation lead to reduced quality of life and, in the worst case, hospitalisation, and eventually sepsis and death [1]. Heavy bacterial colonisation is one of the main reasons for non-healing of chronic wounds, therefore an early detection of an incipient wound infection by the attending physician is crucial for the choice of appropriate treatment to reduce the severity of the disease and avoid the chronicity $[2,3]$. In addition, wounds are often treated with antibiotics prophylactically, leading to unnecessary selection for bacterial resistance. Standard procedures for wound infection detection are time consuming (microbiological tests) or show limited reliability due to the subjective judgement of the classical clinical signs - redness, heat, swelling, and pain [4]. Hence, the medical practitioners are interested in the development of simple point of care testing (PoCT) devices based on the objective evaluation of infection biomarkers.

Myeloperoxidase (MPO: EC 1.11.2.2) is a heme-containing enzyme released by activated neutrophils from the intracellular azurophilic granules. MPO catalyses the oxidation of chloride ions to hypochlorous acid ( $\mathrm{HClO})$ using hydrogen peroxide as cosubstrate. $\mathrm{HClO}$ acid is the most powerful bactericidal oxidant produced by neutrophils (Davies 2011). Besides its antimicrobial activity, MPO plays an important role in the regulation of connective tissue catabolism and degradation, through modifying the proteases/anti-proteases balance [6]. MPO shows about ten times higher activities in 
infected wounds compared to non-infected [7]. Therefore, the detection of this enzyme by PoCT have been postulated as a useful biomarker for wound diagnostics $[7,8]$.

PoCT devices in wound care should deliver fast results while being user friendly, moreover the widespread wound chronicity demands cheap and easily manufactured PoCT devices. In the last decade, chemically patterned paper has showed great potential for the development of versatile microfluidic devices that can be used as PoCT devices [9]. Paper is cheap, biodegradable and biocompatible, its porous cellulose structure wicks fluids without the requirement of a pumping mechanism and is usually white, being the best platform for colorimetric detection [10]. These paper-based microfluidic devices achieve the detection properties by incorporating sensing molecules, e.g. enzyme substrates that are immobilized in specific spots within the patterned paper where they will react when the liquid sample flows through the device [11].

The use of phenolic compounds has been suggested for the colorimetric detection of MPO but their hydrophilic nature impedes their correct immobilisation against a flowing liquid and requires chemical modifications to increase their hydrophobicity $[12,13]$. Moreover, oxidation of phenolic compounds usually yields brownish colours difficult to distinguish from wound fluid colour.

Here, we present a system for MPO detection that can be used in paper-based lateral flow devices. This system is based on the combination of three molecules, the wellknown mediator for oxidative enzymes (ABTS), an oxidizable dye precursor $(m$ phenylenediamine), which yields a clear distinguishable bright purple product, and a cationic polyelectrolyte (branched polyethyleneimine) to achieve immobilisation onto the negatively charged cellulose against the flowing wound liquid. 


\section{Materials and methods}

\section{Substrate mixture preparation and immobilisation}

Polyethyleneimine branched $50 \%$ (PEI) 750000Da, m-phenylenediamine (mPD), pphenylenediamine ( $p P D)$, glucose, 2,2'-azino-bis(3-ethylbenzothiazoline-6-sulfonic acid) diammonium salt (ABTS), dimethyl sulfoxide (DMSO), bovine serum albumin (BSA), Tween ${ }^{\circledR} \quad 20$ and Whatman ${ }^{\mathrm{TM}}$ chromatography $1 \mathrm{CHR}$ paper, Amicon ${ }^{\circledR}$ centrifugal filter units and glucose oxidase (GOX) from Aspergillus niger were purchased from Sigma-Aldrich ${ }^{\circledR}$. Human myeloperoxidase (MPO) was purchased from Planta Natural Products (PNP, Vienna Austria). A wound exudate sample from an infected wound was kindly provided by Dr....from Hospital of Terrassa.

In order to improve the fluidity of enzymes through the Whatman ${ }^{\mathrm{TM}}$ chromatography 1CHR paper, strips were cut $(1.5 \mathrm{~cm} \times 3 \mathrm{~cm})$, soaked for $2 \mathrm{~h}$ at RT in a buffer solution (200 mM sodium phosphate buffer $\mathrm{pH} 6.5$ ) containing $0.5 \%$ BSA and $0.1 \%$ Tween $^{\circledR}$ 20 to and dried overnight at $37^{\circ} \mathrm{C}$. Stocks of compounds $m \mathrm{PD}$ and $p \mathrm{PD}(20 \mathrm{mM})$ were prepared in DMSO. A mixture containing one of the selected phenylenediamines (2 $\mathrm{mM}), \operatorname{ABTS}(2 \mathrm{mM})$, glucose $(2 \mathrm{mM})$ and PEI $(10 \%)$ prepared in $200 \mathrm{mM}$ phosphate buffer $\mathrm{pH} 6.5$ was applied by drop casting $(2.5 \mu \mathrm{l})$ on the centre of the paper strip and dried with air stream.

\section{Visual Detection of MPO immobilized on paper strips}

Hydrogen peroxide, the co-substrate of MPO, was generated in the system by the conversion of glucose with glucose oxidase (GOX) from Aspergillus niger. The GOX solution $(2 \mathrm{mg} / \mathrm{ml})$ was applied by drop casting $(2.5 \mu \mathrm{l})$ on the centre of the paper strip before the immobilised substrate mixture in the sample flow direction, and dried with 
air stream. The oxidation of the immobilised substrate mixture to its coloured product was performed using purified MPO. MPO was diluted in sodium phosphate buffer (200 $\mathrm{mM}, \mathrm{pH} 6.5)$ to different final concentrations . The MPO solution $(60 \mu \mathrm{l})$ was applied at the tip of the paper strip close to the immobilised GOX inducing a liquid flow that first meets and drags GOX and finally encounters the immobilised substrates. When haemoglobin interference was assessed, haemoglobin $\left(\right.$ Sigma-Aldrich $\left.^{\circledR}\right)$ was diluted in sodium phosphate buffer $(200 \mathrm{mM}, \mathrm{pH} 6.5)$ to final concentrations of 0.01 and 0.001 $\mathrm{mM}$ and applied to the paper strip in the same manner as the MPO. In all the cases once the flow passed through the entire length of the strip the incubation time (5 min) at RT was initiated. After the incubation period the colour change was measured using a spectroflash ${ }^{\circledR}$ SF300 with CIE Standard Illuminat D65 according to the CIELab concept. As a reference, a paper strip with immobilized substrate, but without enzyme was used.

\section{Purple Dye separation by HPLC-DAD system}

Prior to the HPLC analysis, the enzymes (MPO and GOX) were removed from the reaction mix through the use of centrifugal filters from Amicon ${ }^{\circledR}$ with molecular weight cut-off of $30 \mathrm{kDa}$. The resulting sample was analysed by HPLC. The dye component was separated and purified by high-performance liquid chromatography (HPLC) coupled with a diode array detector (DAD). The analysis was performed using an Agilent Technologies 1200 HPLC system, consisting of a degasser, a quaternary pump, and an automatic sample injector. The system also includes a diode array detector for recording the absorption spectra of the samples. A Microsorb-MV C-18 reserved-phase column $(150 \times 4.6 \mathrm{~mm}, 5 \mu \mathrm{m}$ particle size $)$ was used to perform the chromatographic separation. The flow rate was $1 \mathrm{~mL} / \mathrm{min}$ and the purple dye was detected at $550 \mathrm{~nm}$. Gradient elution was carried out using MilliQ water as solvent A and methanol as 
solvent B. During the analysis, the solvent gradient was programmed as: $0-8 \mathrm{~min}$ : $85 \% \mathrm{~A} / 15 \% ; 8-15 \mathrm{~min}: 50 \% \mathrm{~A} / 50 \% \mathrm{~B} ; 15-20 \min : 40 \% \mathrm{~A} / 60 \% \mathrm{~B} ; 20-25 \mathrm{~min}:$ $30 \% \mathrm{~A} / 70 \% \mathrm{~B} ; 25-30 \min : 25 \% \mathrm{~A} / 75 \% \mathrm{~B} ; 30-40 \min : 50 \% \mathrm{~A} / 50 \% \mathrm{~B}, 40-50 \mathrm{~min}:$ $15 \% \mathrm{~A} / 75 \% \mathrm{~B}$. With column cleaning and equilibrium steps, the total run time for each sample was $50 \mathrm{~min}$. Agilent ChemStation software was used for data acquisition and processing. No interfering peaks were found in the chromatographic separation at the retention time. A fraction collector was used to collect the fractions corresponding to the dye component in the sample. Afterwards the collected fractions were analysed using ESI-TOF.

\section{Analysis of the MPO-mediated oxidation products through ESI-TOF}

Mass data of the MPO-mediated oxidation products purified by HPLC system was obtained by electrospray ionization in positive mode coupled with a time-of-flight mass spectrometry (ESI TOF MS). The equipment used was a Micro Tof-Q Instrument (Bruker Daltonics GbmH, Bremen, Germany), interfaced with a Series 1200 HPLC Agilent pump, equipped with an auto-sampler, which was controlled using the Compass software. The carrier buffer was a $1 \% \mathrm{HCOOH}$ water solution and the injection performed with a LC system without column. The samples were injected under the following conditions: desolvation temperature $180{ }^{\circ} \mathrm{C}$, capillary-counter electrode voltage $5 \mathrm{kV}$, dry gas $6 \mathrm{~L} / \mathrm{min}$. Spectra was collected throughout an $\mathrm{m} / \mathrm{z}$ range from 50 to $3000 \mathrm{~m} / \mathrm{z}$. The electrosprayed solutions were prepared following the same ratio mixture used in the paper device. Due to a limitation of the mass detection system, the analysis of the PEI molecular mass was performed with a PEI with a lower molecular weight (but same ramification degree) than the one used for the immobilisation on the 
paper strips (800 vs $750000 \mathrm{Da}$ ) assuming that any MPO oxidising reaction occurring with a PEI of 750000Da would occur with a smaller one.

\section{Results and discussion}

Fast diagnosis of wound infection status is crucial to implement proper treatment and avoid chronic infections. Significant differences in MPO activity between infected and non-infected wound fluids revealed the potential of this enzyme as biomarker for infection detection in wounds [7]. The immobilisation of MPO substrates that once oxidised change their colour is the most obvious strategy to follow in the development of PoCT for MPO detection. Hence, we developed a new MPO detection system, based on enzyme-catalysed oxidative synthesis of a dye, which can be easily incorporated on paper-based PoCT devices.

In the context of a new MPO detection system that could be applied for a PoCT wound diagnostic device, a clear colour change distinguishable from the wound fluid colour background is required. In this sense, we referred to commonly used polymeric dyes for hair based on phenylenediamines. Oxidation of these molecules by laccases yielded chromogenic products with distinguishable bright colours (purple and violet) (Saito et al. 2012). The higher reduction potential of MPO compared to laccases strongly encouraged the assessment of these compounds as chromogenic MPO substrates (Davies 2011; Jones \& Solomon 2015).

The three isomers $p$-phenilenediamine $(p \mathrm{PD}), m$-phenilenediamine $(m \mathrm{PD})$ and $o$ phenilenediamine $(\mathrm{OPD})$ were screened for oxidation by MPO resulting in products with black, pale brown and yellow colours respectively (Fig 1). Surprisingly, when the mediator ABTS was added in the reaction mixture the MPO catalysed oxidation of $p$ PD and $m$ PD resulted in blue and purple respectively. These colours, clearly distinguishable 
from wound fluid colours, revealed the suitability of substrate pairs (phenylenediamines and ABTS) for the development of a MPO detection system (Fig. 1). .

The applicability of the new MPO detection system was tested immobilising the substrate pairs on paper strips as a model of paper-based diagnostic device. Immobilisation was achieved by the addition of the polycationic polymer PEI in the substrate pair mixture that was applied and dried onto the surface of the paper strips. The immobilised substrate combinations were assessed for their ability to detect MPO, flowing through the paper strip a solution with different MPO concentrations as well as infected wound exudates.

The immobilised combination composed of $m \mathrm{PD}$, ABTS and PEI (M-A-P) reacted to the MPO levels described for infected wound fluid [7] showing a fast colour change to bright purple. Meanwhile, the lack of colour change to the naked eye with the noninfected MPO levels revealed the M-A-P combination suitable for detection of infected wound fluid avoiding a wrong detection of the infection (Fig. 2). On the other hand, the combination comprised of $p \mathrm{PD}, \mathrm{ABTS}$ and $\mathrm{PEI}(\mathrm{P}-\mathrm{A}-\mathrm{P})$ showed low reactivity revealing a faint colour change to blue upon all the tested MPO levels (Fig 2). Due to the peroxidase activity of haemoglobin, cross-reaction was evaluated with the concentrations of this protein present in infected and non-infected wound fluids [12]. For both substrate combinations, cross-reaction only occurred with the haemoglobin levels of infected wound fluid. In the case of the M-A-P combination, the slow cross reaction resulted in a faint colour change not comparable to the colour change induced by the MPO concentrations of infected wound fluids. On the other hand, the crossreaction colour induced by haemoglobin on the $\mathrm{P}-\mathrm{A}-\mathrm{P}$ combination is equivalent to 0.23 $\mu \mathrm{M}$ of MPO. Therefore, out of both assessed combinations, M-A-P proved optimum for MPO detection. 



\section{Role of ABTS in the MPO oxidation of $m$ PD}

It was striking to observe that the use of ABTS in the MPO oxidation of $m$ PD yielded a coloured product with different chromogenic properties than in the absence of the mediator. Identification of the purple product was performed in order to determine the role of $\mathrm{ABTS}$ in the oxidation of $\mathrm{mPD}$. Liquid chromatography allowed the isolation of a purple coloured product with absorbance at $550 \mathrm{~nm}$ (Fig. 3, A and B). Mass spectrometry of the isolated purple dye revealed a mass of $619 \mathrm{~m} / \mathrm{z}$ confirming a single stable reaction product of the MPO mediated oxidation of ABTS and $m$ PD (Fig. 3, C). Both, the detected mass and isotopic distribution allowed to determine the empirical formula of the purple molecule $\left(\mathrm{C}_{24} \mathrm{H}_{22} \mathrm{~N}_{6} \mathrm{O}_{6} \mathrm{~S}_{4}\right)$ which agrees with ABTS covalently bound to $m$ PD. Based on previously reported reaction between ABTS radical and tyrosyl radical protein residues to form a purple product [17] we suggest a similar reaction. A molecule of $m$ PD reduces the ABTS radical, produced by MPO, resulting in $m$ PD radical. This molecule subsequently reacts with another ABTS radical forming a stable purple mPD-ABTS conjugate. According to this suggested mechanism and the determined empirical formula of the purple dye, we propose that the mPD-ABTS conjugated is formed via the reaction shown in Figure 4. Therefore, ABTS acts as mediator in the oxidation of $m \mathrm{PD}$ but also as a reaction substrate for the synthesis of the purple dye.

\section{Interaction between MPO coloured products and PEI}

The use of the polycation PEI provided an easy route for immobilisation of the MPO substrate pairs (phenylenediamines and the mediator ABTS) on the paper strips. In order to determine if the immobilisation mechanism of PEI with the other substrates is through a MPO-catalysed oxidative cross-linking, PEI mass analysis for both MPO 
oxidised and non-oxidised M-A-P combination was conducted. In both cases the obtained mass profile was the same (data not shown), indicating a no-covalent interaction and suggesting an electrostatic interaction. In fact, nanofibrous membranes containing PEI has been utilised as adsorbent for removal of anionic azo dyes based on the electrostatic interactions between the PEI-containing nanofibrous membrane and the anionic dyes detected by x-ray photoelectron spectroscopy [18]. It is plausible that similar interactions can occur between the purple ABTS-mPD conjugate due to its similar to azo dyes structure presenting negatively charged sulfonic groups. 


\section{Conclusions}

Here we report on a new MPO detection system based on MPO-catalysed oxidative synthesis of a dye, which bright purple colour does not interfere with the colour of the wound fluid background. The detection system can be easily incorporated in paperbased lateral flow device, without any chemical modification of the detection path, through the application of the polycation PEI. Fast visual detection of MPO levels corresponding to infected wounds was feasible via a distinctive colour change, while avoiding cross-reaction with haemoglobin. Liquid chromatography and mass spectroscopy allowed the identification of the bright purple dye product derived from covalent interaction of ABTS radical and $m$ PD. The successful implementation of this MPO detection system opens the door to its applicability in the development of cheap PoCT devices for wound infection. 


\section{Acknowledgements}

This work was supported by the European project "Multiplex point-of-care device for lung disease biomarkers in sputum" (PCIN-2016-134).

Declarations of interest: none 


\section{References}

[1] R.G. Frykberg, J. Banks, Challenges in the Treatment of Chronic Wounds., Adv. Wound Care. 4 (2015) 560-582. doi:10.1089/wound.2015.0635.

[2] K.F. Cutting, R.J. White, Criteria for identifying wound infection--revisited., Ostomy. Wound. Manage. 51 (2005) 28-34.

[3] R.G. Sibbald, H. Orsted, G.S. Schultz, P. Coutts, D. Keast, International Wound Bed Preparation Advisory Board, Canadian Chronic Wound Advisory Board, Preparing the wound bed 2003: focus on infection and inflammation., Ostomy. Wound. Manage. 49 (2003) 24-51.

[4] S.E. Gardner, R.A. Frantz, B.N. Doebbeling, The validity of the clinical signs and symptoms used to identify localized chronic wound infection, Wound Repair Regen. 9 (2001) 178-186. doi:10.1046/j.1524-475x.2001.00178.x.

[5] M.J. Davies, Myeloperoxidaseederived oxidation: mechanisms of biological damage and its prevention, J. Clin. Biochem. Nutr. JCBN J. Clin. Biochem. Nutr. 48 (2011) 8-19. doi:10.3164/jcbn.111006FR.

[6] G.J. Peppin, S.J. Weiss, Activation of the endogenous metalloproteinase, gelatinase, by triggered human neutrophils., Proc. Natl. Acad. Sci. U. S. A. 83 (1986) 4322-6.

[7] a Hasmann, E. Wehrschuetz-Sigl, a Marold, H. Wiesbauer, R. Schoeftner, U. Gewessler, a Kandelbauer, D. Schiffer, K.P. Schneider, B. Binder, M. Schintler, G.M. Guebitz, Analysis of myeloperoxidase activity in wound fluids as a marker of infection., Ann. Clin. Biochem. 50 (2013) 245-54. doi:10.1258/acb.2011.010249.

[8] G. Tegl, D. Schiffer, E. Sigl, A. Heinzle, G.M. Guebitz, Biomarkers for infection: enzymes, microbes, and metabolites, Appl. Microbiol. Biotechnol. 99 (2015) 
4595-4614. doi:10.1007/s00253-015-6637-7.

[9] N.A. Meredith, C. Quinn, D.M. Cate, T.H. Reilly, J. Volckens, C.S. Henry, A. Birbas, I. Ben-Yaacov, K.W. Plaxco, G.M. Whitesides, Paper-based analytical devices for environmental analysis, Analyst. 141 (2016) 1874-1887. doi:10.1039/C5AN02572A.

[10] P. Shah, X. Zhu, C. Li, Development of paper-based analytical kit for point-ofcare testing, Expert Rev. Mol. Diagn. 13 (2013) 83-91. doi:10.1586/erm.12.130.

[11] A.K. Yetisen, M.S. Akram, C.R. Lowe, E. Carrilho, T.M. Swager, A. Ozcan, F. Zeng, M. Prentiss, G.M. Whitesides, P. V. Baptista, K.P. Derose, M. V. Shea, C.M. Beighley, C.A. Dahl, J. Wasserman, J. Cobbing, R. Calow, C. Hunt, A. Hussain, M.C. Acreman, J. King, S. Malomo, E.L. Tate, D. O’, S. Milner, I. Steyl, Paper-based microfluidic point-of-care diagnostic devices, Lab Chip. 13 (2013) 2210. doi:10.1039/c31c50169h.

[12] D. Schiffer, G. Tegl, R. Vielnascher, H. Weber, R. Schoeftner, H. Wiesbauer, E. Sigl, A. Heinzle, G.M. Guebitz, Fast Blue RR-siloxane derivatized materials indicatewound infection due to a deep blue color development, Materials (Basel). 8 (2015) 6633-6639. doi:10.3390/ma8105329.

[13] D. Schiffer, G. Tegl, R. Vielnascher, H. Weber, A. Herrero-Rollett, E. Sigl, A. Heinzle, G.M. Guebitz, Myeloperoxidase-responsive materials for infection detection based on immobilized aminomethoxyphenol, Biotechnol. Bioeng. 9999 (2016) 1-8. doi:10.1002/bit.26025.

[14] K. Otsuka Saito, R. Ikeda, K. Endo, Y. Tsujino, M. Takagi, E. Tamiya, Isolation of a novel alkaline-induced laccase from Flammulina velutipes and its application for hair coloring, J. Biosci. Bioeng. 113 (2012) 575-579. doi:10.1016/j.jbiosc.2012.01.001. 
[15] M.J. Davies, Myeloperoxidaseederived oxidation: mechanisms of biological damage and its prevention, J. Clin. Biochem. Nutr. JCBN J. Clin. Biochem. Nutr. 48 (2011) 8-19. doi:10.3164/jcbn.111006FR.

[16] S.M. Jones, E.I. Solomon, Electron transfer and reaction mechanism of laccases, Cell. Mol. Life Sci. 72 (2015) 869-883. doi:10.1007/s00018-014-1826-6.

[17] B. Åkerström, G.J. Maghzal, C.C. Winterbourn, A.J. Kettle, The Lipocalin $\alpha_{1}$ Microglobulin Has Radical Scavenging Activity, J. Biol. Chem. 282 (2007) 31493-31503. doi:10.1074/jbc.M702624200.

[18] V. Panic, S. Seslija, A. Nesic, S. Velickovic, Adsorption of azo dyes on polymer materials, Hem. Ind. 67 (2013) 881-900. doi:10.2298/HEMIND121203020P. 


\section{Figure captions}

A)

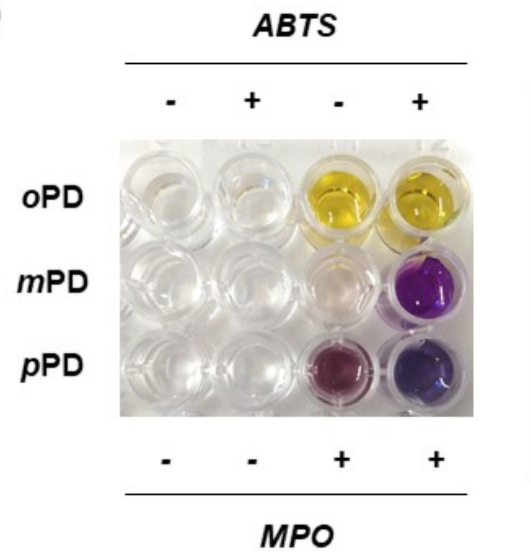

B)

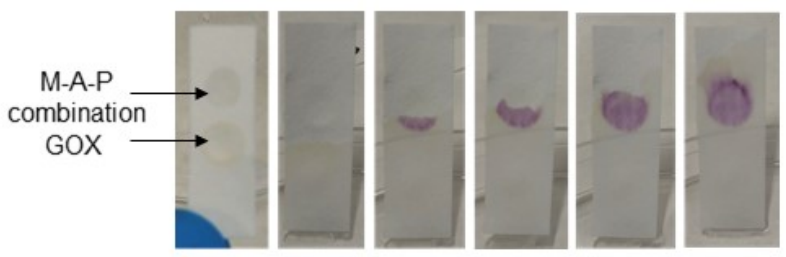

Figure 1: MPO detection by oxidation of phenilenediamines. Panel A corresponds to the evaluation of MPO-catalysed polymeric dye synthesis of $o$ PD, $m P D$ and $p$ PD with $(+)$ and without (-) the mediator ABTS. Panel B shows the visual detection of MPO with M-A-P combination immobilised on a paper strip.
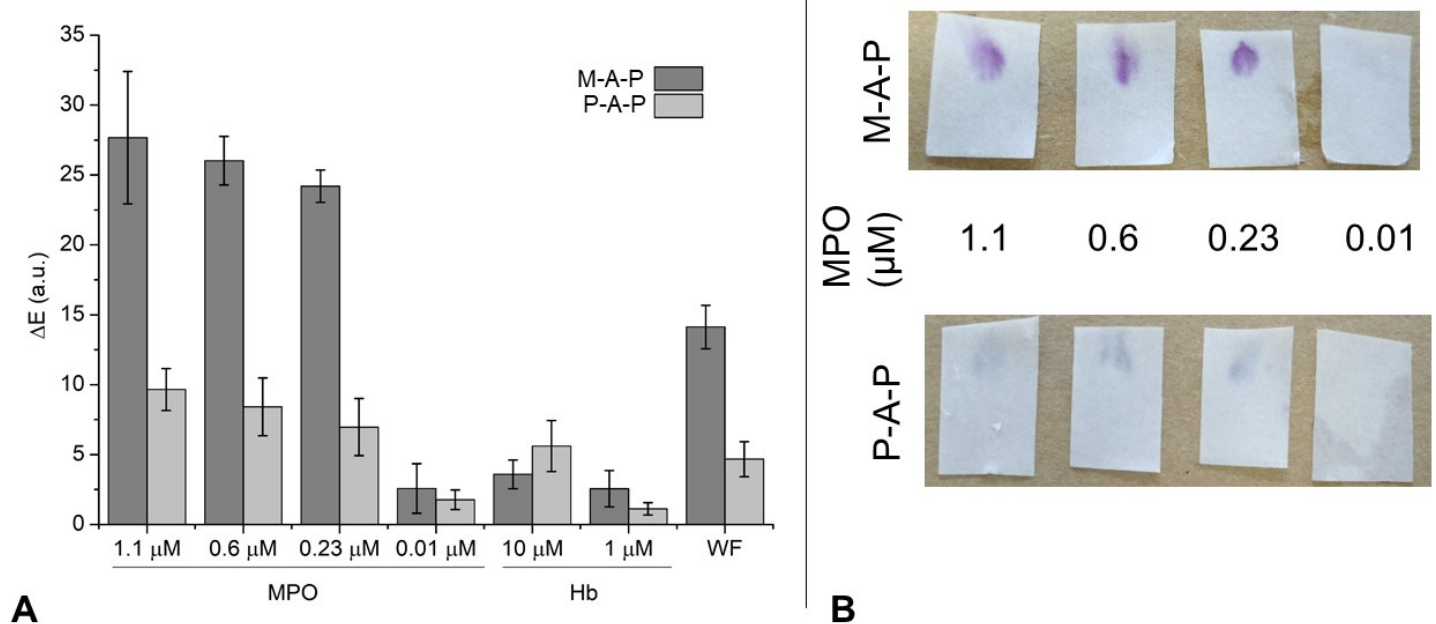

Figure 2: Colour formation of the immobilised compound combinations under different levels of MPO flowed through the paper strip. Panel A shows the colour change of the M-A-P and P-A-P combinations upon oxidation by MPO, haemoglobin $(\mathrm{Hb})$ and infected wound exudate (WF). In Panel B images of the observed colour evolution upon application of different concentrations of MPO are shown. 


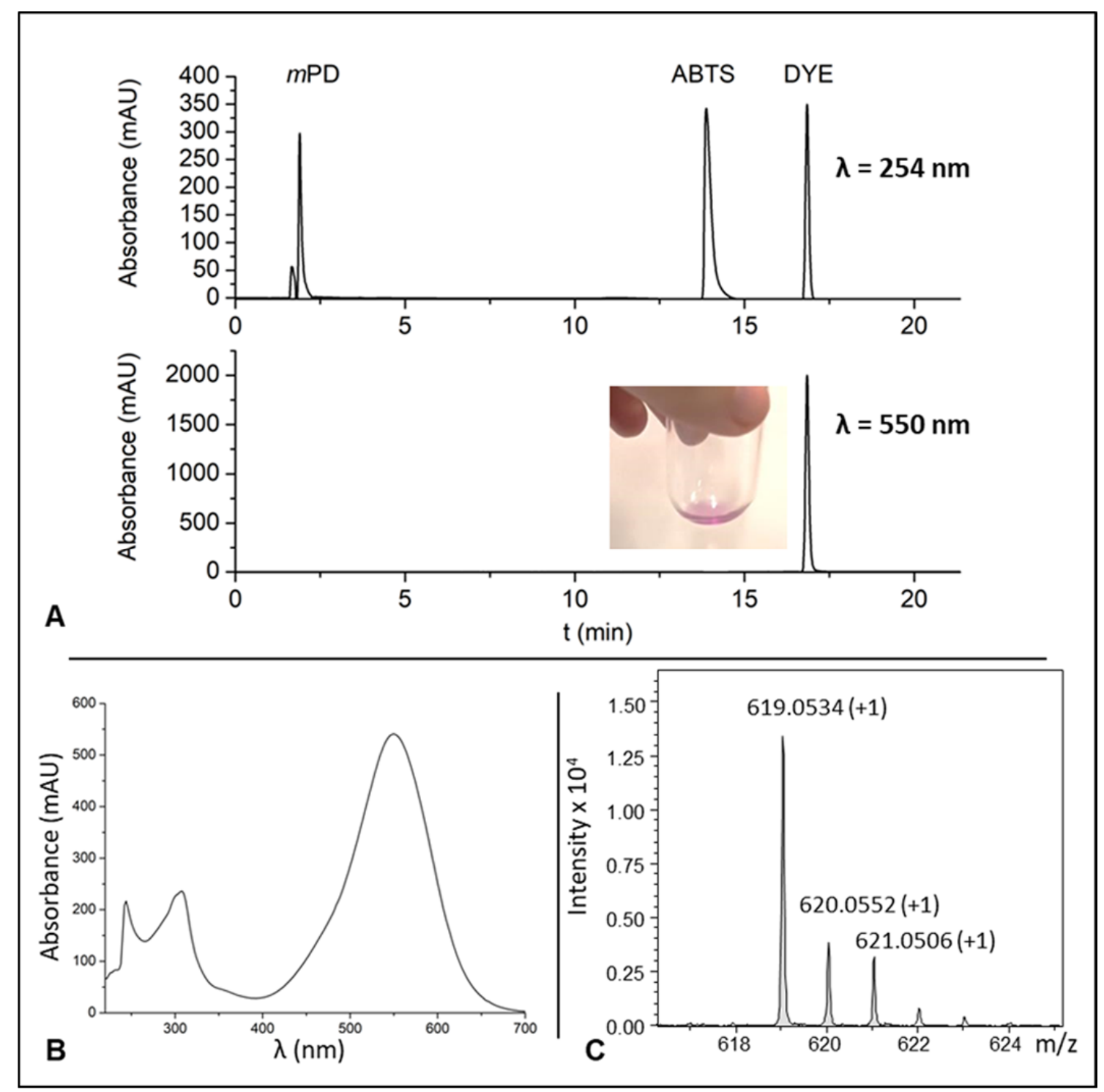

Figure 3: Chromatographic separation and ESI-TOF characterisation of the MPO oxidation products. Panel A shows HPLC-DAD chromatograms obtained at wavelength of 254 and $550 \mathrm{~nm}$. Panel B shows UV-Vis spectra corresponding to the peak at $16 \mathrm{~min}$ of retention time (purple dye). Panel C corresponds to the ESI-MS spectra of the purified purple dye. 


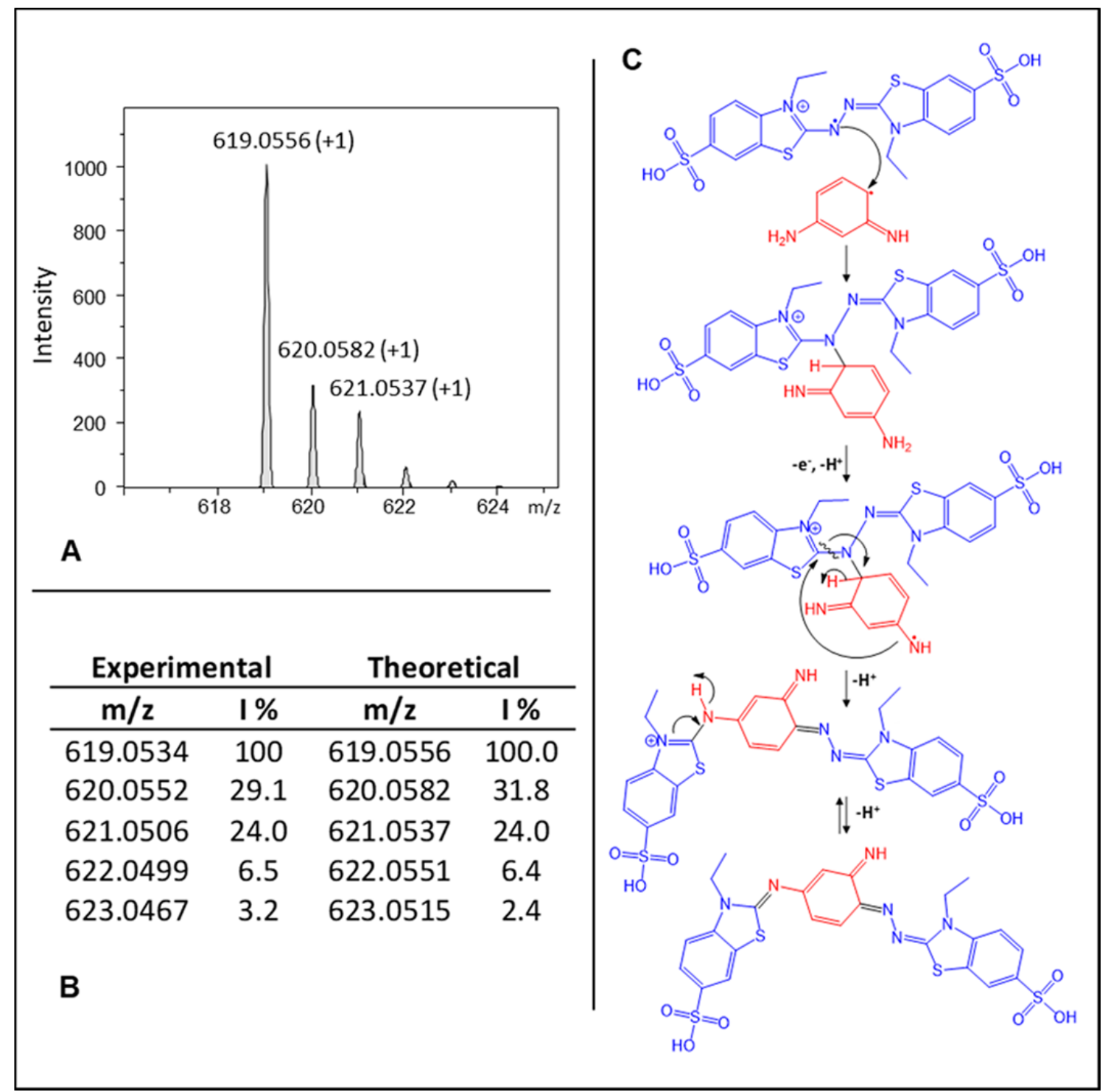

Figure 4: Mass simulation and synthesis mechanism of the purple ABTS-mPD conjugate. Panel A shows theoretical isotopic distribution of $\mathrm{C}_{24} \mathrm{H}_{22} \mathrm{~N}_{6} \mathrm{O}_{6} \mathrm{~S}_{4}$. Panel $B$ shows comparison between the experimental $\mathrm{m} / \mathrm{z}$ peaks and its intensities for the purified purple dye with the theoretical values corresponding to the modelled molecule $\mathrm{C}_{24} \mathrm{H}_{22} \mathrm{~N}_{6} \mathrm{O}_{6} \mathrm{~S}_{4}$. Panel $\mathrm{C}$ shows tentative mechanism of the reactions between radicals $m \mathrm{PD}$ and ABTS yielding the purple ABTS- $m$ PD conjugate. 